\title{
Detection of Industrial Machine Work Errors using LVQ Neural Network
}

\author{
Muh. Rafli Rasyid ${ }^{1}$, Zulkifli Tahir ${ }^{2}$, Syafaruddin ${ }^{3}$ \\ ${ }^{1,3}$ Department of Electrical Engineering, Faculty of Engineering, Universitas Hasanuddin \\ Makassar, \\ Indonesia. 92119 \\ ${ }^{2}$ Department of Informatics, Faculty of Engineering, Universitas Hasanuddin Makassar, \\ Indonesia. 92119 \\ \{rasyidmr17d@student.unhas.ac.id¹, zulkifli@unhas.ac.id², syafruddin@unhas.ac.id³
}

\begin{abstract}
In the world of industry, the utilization of technology machinery industry is one of the most important factors to facilitate the employment of human. However, an industrial machine does not work regardless of fault that can inhibit the production process and cause harm to the industry. This paper aims to detect errors with the industrial machine work to analyze the movement of industrial machinery in a video, at this stage of the process of preprocessing, image resizes, do segmentation method thresholding, and the morphological operations with the opening operation. The further step, the feature extraction performed by converting a binary image into vector data is used as input data in the classification process using Algorithm Learning Vector Quantization (LVQ) Neural Network version 1 and version 2 . Research results obtained detection accuracy reached $100 \%$ for training using LVQ1 much higher than the results of the training using LVQ2 with an accuracy of only $67.59 \%$.
\end{abstract}

Keywords: Computer Vision, Image Processing, Learning Vector Quantization (LVQ) Neural Network.

\section{Introduction}

The industrial world in recent years has shown significant progress; some modern companies have begun to utilize industrial machinery technology by applying artificial intelligence [1]. The existence of these machines is beneficial because it can streamline production time to increase productivity [2] so that more work can be completed. However, a machine can not be separated from work errors that can hinder the production process and cause a large loss for the company [3]. Computer vision will be needed as a solution to the problem, one concept of computer vision is a detection system where a computer with the help of a camera is expected to be able to retrieve data information [4]. In the form of machine works, the preprocessing, segmentation, extraction features and classification to detect normal or abnormal machine motion are the several factors and indicators of work errors in industrial machinery.

Several studies with the concept of computer vision and the use of classification algorithms using learning vector quantization (LVQ) neural networks in various fields have been carried out. Wang et al. Conducted research using the concept of computer vision in detecting wind 
turbine fires using learning vector quantization neural network algorithms to analyze the flames of wind turbines based on color characteristics, area changes, centroid mobility characteristics, and flame circularity. From the results of the research, the accuracy of recognition of features was around 93\%, effectively representing local features of fire [5].

Research with a computer vision-based approach to classification of brain and breast images has been presented. This research used a normalized image for the preprocessing stage. Morphological operations were carried out using erosion, dilation and anisotropic diffusion filters (ADF). Level of co-occurrence matrix (GLCM) and LVQ NN for normal and abnormal detection classifications. The results of the proposed system were evaluated by three performance parameters, namely sensitivity, specificity, and accuracy. The proposed method achieves a sensitivity of $82.50 \%$, specificity of $42.86 \%$ and accuracy of $68.85 \%$ for breast cancer database, while for clinical Brain MRI database sensitivity results are $81.82 \%$, specificity $77.53 \%$ and accuracy of $79.35 \%$.

Research on computer vision was also conducted to detect areas affected by skin diseases or called Vitiligo with the help of images taken by the camera and classify the affected area [6], the implementation of LVQ neural networks provided good accuracy of $92.22 \%$ and kappa values 0.810 .

In contrast, conducting computer vision-based research related to the identification of arteries for use in biometric systems [7]. The results of the researchers explain that each has a different vein shape and has a unique, in feature extraction using the symmetry phase method to separate arteries with other parts of the palm. 470 samples of arterial images are used for the identification stage using the method LVQ NN classification and obtained results of accuracy reaching $94 \%$ of the total sample [8]. Meanwhile, a computer vision based research on determining the range of apple prices by classifying apple colors, using LVQ NN to divide apples into three levels, and experimental results showed NN LVQ accuracy reached 93\%, which was slightly higher than SVM 92.3\% [9].

The process of processing information from images in the concept of computer vision can be divided into three stages, namely preprocessing, feature extraction and classification/ identification. This research will also follow these three stages. First, preprocessing is done by changing the size of the image to be smaller so that it can reduce memory usage and speed up the execution process, then segmenting is done by changing the RGB image into a binary image with the thresholding method and performing morphological operations to reduce image noise; Second, conducting feature extraction by converting binary images into vector data; Third, identification by classifying the characteristics of vector data using the LVQ NN learning vector algorithm so that the network architecture is obtained, the training results are stored and used for the testing phase, the results of the detection are reviewed to determine the detection success rate.

\section{Materials and Method}

The system description proposed in this research can be seen in Figure 1 below: 


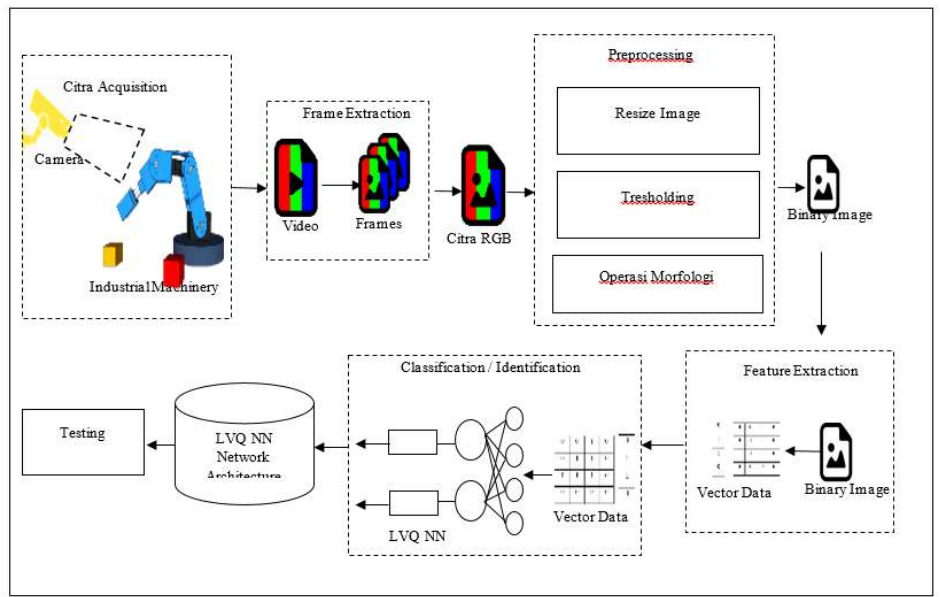

Fig 1. System Architecture

\subsection{Data Description}

In this paper, the acquisition of industrial machine motion video image was taken using a 15 MP resolution camera as input data with a size of $864 \times 480$ pixels, the data used in the form of two video movements namely industrial machine movement when working correctly with 24 seconds duration and industrial machine movement video on when working in a wrong state with a duration of 51 seconds, then an extrusion frame was carried out resulting in 1145 training data frames consisting of 371 data for correct motion and 774 data for wrong motion, while the test data used was 600 data consisting of 250 correct motion data and 350 data wrong movement data. The data retrieval process is done by placing the camera in a static position as far as $50 \mathrm{~cm}$ from the object; in this paper, the object used is industrial machine prototype in the form of a robotic arm.

\subsection{Preprocessing}

Pre-processing is the first process in image processing, at this stage do some changes to the original image to simplify the process at a later stage, the first stage first performed in the preprocessing is to reduce the image size of $10 \%$ of the value of the original image, and then change the RGB image into a grayscale image with the following equation [10]:

$$
y=0,299 R+0.587 G+0.144 B
$$

The next step is to change the grayscale image into a binary image with thresholding method, in this paper thresholding value determined at $128 / 256$ or 0.5 , while the equation to change an advanced grayscale image binary image can be seen in equation [10]:

$$
y^{\prime}=\left\{\begin{array}{l}
1, y \geq \text { treshold } \\
0, \text { otherwise }
\end{array}\right.
$$


The binary image usually results thresholding method still has a lot of noise, to eliminate noise in the images used morphological operations such as opening area, image morphology operation equation shown in equation [10]:

$$
A^{\circ} B=A \ominus B \oplus B
$$

All proceeds from the stages of processing can be seen in Figure 2:

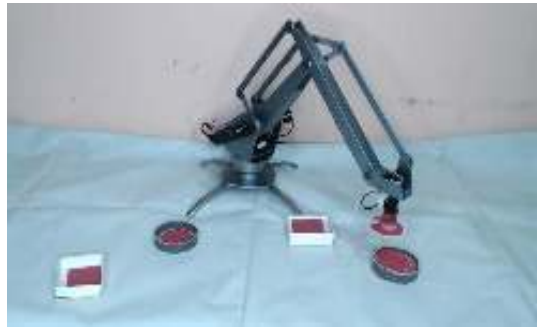

(a)

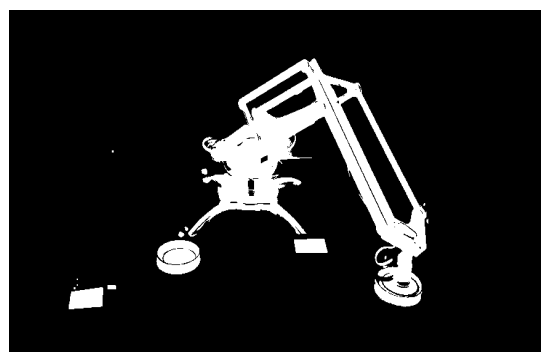

(c)

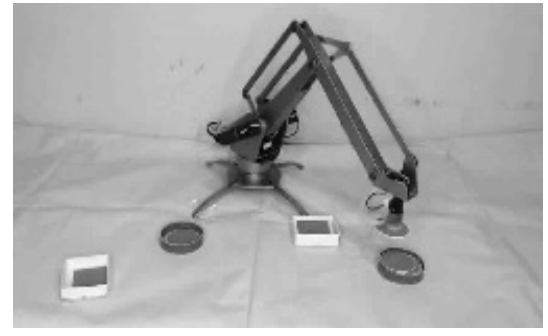

(b)

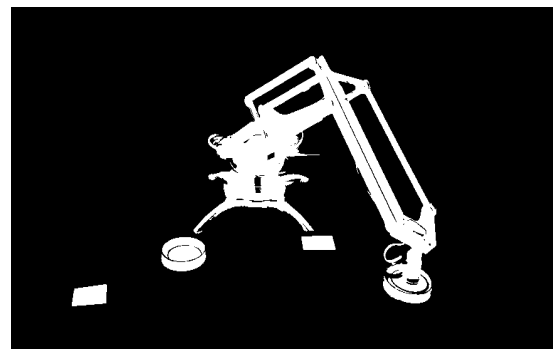

(d)

Fig 2. Preprocessing: (a) original image, (b) grayscale image (c) binary image (d) binary image with morphological operations

\subsection{Feature extraction}

Feature extraction is the process of selecting the unique characteristics of data to be processed. this stage image preprocessing results in the form of binary image has a size of $48 \mathrm{x}$ 87 matrix is converted to vector matrix of $4176 \times 1$, then to get the characteristic value vector coupled with the total number of data that as many as 1145 frames get a new matrix size of 4176 $\mathrm{x} 1145$, then the new matrix is used as input for training using LVQ NN.

\subsection{Classification and Identification}

At this stage, the data vector generated from feature extraction is used as input data that is processed so that a class is obtained. At this stage, the training process and testing process will be carried out using the LVQ1 and LVQ2 algorithms, will do the training process and the testing process using algorithms LVQ1 and LVQ2, 
The training process is done using training data that has been extracted earlier to distinguish between the motion of one with the correct motion is then evaluated to know the value of accuracy by comparing the output and the target, the next process is a testing process, in this process the data generated from the training will be used to map test data to obtain the output data, and then labeled.

\subsection{Lvq Neural Network Model}

Learning vector quantization neural network is one of the methods of artificial neural network that includes one of the unsupervised competitive learning algorithms of algorithms Kohonen Self-Organizing Map (SOM). LVQ NN has three main neuron layer, the input layer, hidden layer, and output layer [11] [12],

This algorithm has the objective to obtain the closest value to the class division of vector to minimize the errors in the classification process, every part of the output of the algorithm LVQ NN declare a class that previously has been determined, there are two types of LVQ NN algorithms for classification and vector data that is LVQ1 LVQ2 [13], On LVQ1 only one neuron that can be refurbished and set which is the nearest candidate of the target or called neurons winner[14], while the runner up LVQ2 introduce neurons, in which the weight vector neurons and neuron runner-up winners will be updated. However, in its application, the LVQ1 algorithm is more widely used than LVQ2 [9]. The model of LVQ NN in general can be seen in Figure 3.

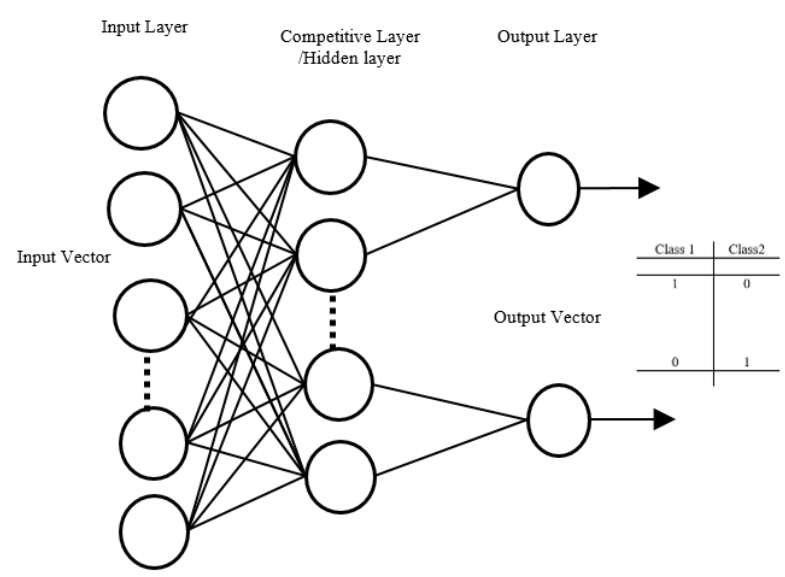

Fig3. Structure of LVQ NN

LVQ algorithm has a basic concept to measure the distance of the nearest input vector with competitive neurons [15]. If the class input vector equal to that of the output layer neurons, neurons that is closest in the competitive layer will move in the direction of the input vector; if not then move in the opposite direction. 


\subsection{Performance}

To evaluate the detection accuracy of the calculation using the following equation:

$$
\text { accuracy }=\frac{\text { number of correct detection }}{\text { total amount of data }} * 100
$$

\section{Result and Discussion}

This section will describe some of the work done on research covering the processing of video data acquisition, image processing, training, and testing using LVQ NN algorithms as well as evaluation of the detection accuracy of measurement results obtained based on the parameters set by LVQ NN. The acquisition of video data captured by the camera $15 \mathrm{MP}$ resolution camera as $864 \times 480$ pixels then extracted the frame which produced 1145 frames of training data and 600 test data.

The processing of images carried out in this paper was a preprocessing process in which RGB images were converted into grayscale images, then continued by changing the grayscale image into a binary image, the result of a binary image is noise and to eliminate noise in the image morphology operation is carried out using the method of opening area.

Furthermore, the second stage in the image processing is feature extraction, feature extraction done by changing the matrix binary image into a matrix-vector, which is used as a data input to the training and testing for identification.

The identification process in this paper using algorithms LVQ NN, some parameter is set to conduct training The first phase is to determine the target, where the target is divided into two classes: class 1 for true conditions, and class 2 for false conditions, the second stage is to determine the value of the parameter LVQ NN is the number of hidden layers, the maximum number of iterations, and learning rate.

In this paper, the number of hidden layers is 10,50,100, 200, 300, 400 and 500, which will be tested at the maximum iteration 10 iteration and learning rate of 0.01 . Results of testing LVQ NN parameter values can be seen in Table 1.

Table 1. LVQ Accuracy Parameters

\begin{tabular}{|c|c|c|c|c|c|c|c|}
\hline \multicolumn{4}{|c|}{ LVQ1 } & \multicolumn{4}{|c|}{ LVQ2 } \\
\hline Hidden Layer & Iteration & Ex. Time & Akurasi \%) & Hidden Layer & Iteration & Ex. Time & Akurasi \% \\
\hline 10 & 1 & 00:08 & 100 & 10 & 10 & 00:06 & 67.59 \\
\hline 100 & 2 & $02: 23$ & 100 & 100 & 10 & $09: 15$ & 67,59 \\
\hline 200 & 3 & $05: 52$ & 100 & 200 & 10 & 19:07 & 67.59 \\
\hline 300 & 4 & $12: 17$ & 100 & 300 & 10 & $28: 37$ & 67.59 \\
\hline 500 & 6 & $29: 07$ & 100 & 500 & 10 & 49:19 & 67.59 \\
\hline Avg & & & 100 & & & & 67.59 \\
\hline
\end{tabular}

From the results of Table I, it can be seen that the change in the number of hidden layers used in the training process only affects the execution time, but does not affect the detection accuracy value, while the value of accuracy obtained up to $100 \%$ using LVQ 1, much higher than the results of the training using LVQ2 with an accuracy of only $67.59 \%$. Then the network architecture results from the training are stored for use at the testing stage of the test image. 
The testing process is done the same image processing with image processing in the training process, from the preprocessing stage to the stage of feature extraction, vector data results of the test data have been extracted used as input into LVQ NN network architecture that has been built during the training. The image of the test results then labeled according to the conditions the final weights for each class in the form of a red-cross sign as any condition or results of 1 st class output and a green check mark to the true condition or resulted output to class 2 . The image that has been labeled can be seen in Figure 4.

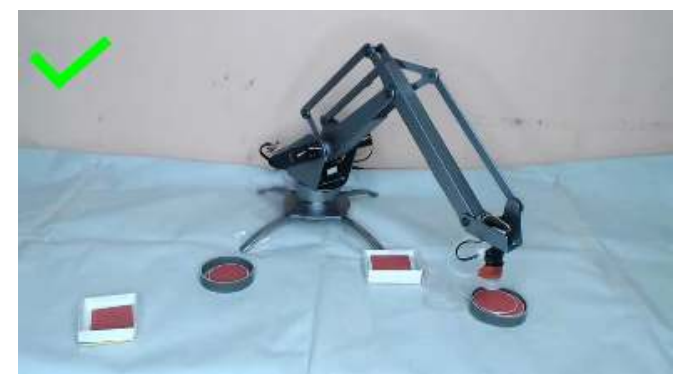

(a)

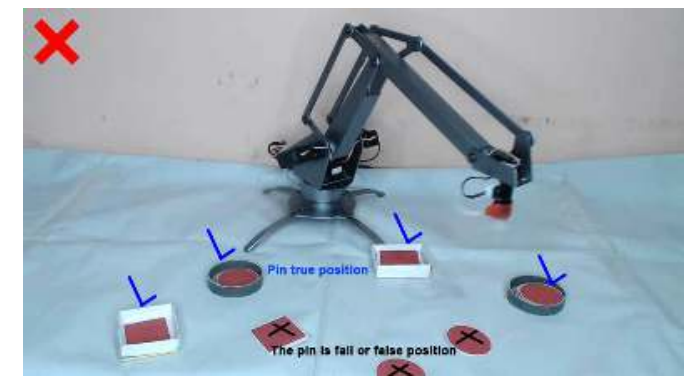

(b)

Fig 4. Results of testing: (a) the correct conditions, (b) false condition

Figure 4 (b) shows the false condition of the industrial machine that was successfully detected; it can be seen that some pins or objects that want to be moved by the machine are not placed in the appropriate place, or the pins fall during the transfer process. Figure 4 (a) shows the placement of pins/objects that want to be moved by a machine that placed correctly or no pins/objects dropped.

\section{Conclusion}

In the paper, researchers implement image processing as a representation of the concept of computer vision algorithms using learning vector quantization neural network for fault detection engines work industry, preprocessing is done to change the image so that it is easier to use on the next stage, the stage of feature extraction is done by making a change binary image into vector data, then the data vector are trained using the algorithm LVQ1 and LVQ2, the detection results are evaluated to generate value detection accuracy, while the results of each of $100 \%$, for training LVQ1 higher than the training results using LVQ2 with only $67.59 \%$.

\section{References}

[1] A. G. Frank, L. S. Dalenogare, and N. F. Ayala, "Industry 4.0 technologies: Implementation patterns in manufacturing companies," Int. J. Prod. Econ., vol. 210, pp. 15-26, 2019.

[2] G. Erdoğan, "Land selection criteria for lights out factory districts during the industry 4.0 process," J. Urban Manag., no. July 2018, pp. 1-9, 2019.

[3] Zulkifli Tahir, "A Hybrid Maintenance Management Model in Decision Support System for Small and Medium Food Processing Industries," Universiti Teknikal Malaysia Melaka, 2010. 
[4] M. Tiwari, S. S. Lamba, and B. Gupta, "An image processing and computer vision framework for efficient robotic sketching," Procedia Comput. Sci., vol. 133, pp. 284289, 2018.

[5] C. Wang, L. Sun, T. Yuan, and X. Sun, "Wind turbine fire image detection based on LVQ Neural Network," 6th Annu. IEEE Int. Conf. Cyber Technol. Autom. Control Intell. Syst. IEEE-CYBER 2016, pp. 437-441, 2016.

[6] A. Anthal, Jyotsna; Upadhyay, Anand; Gupta, "Detection of V itiligo S kin D isease using LVQ Neural Network," 2017 Int. Conf. Curr. Trends Comput. Electr. Electron. Commun., pp. 922-925, 2017.

[7] R. Sonavane, P. Sonar, and S. Sutar, "Classification of MRI brain tumor and mammogram images using learning vector quantization neural network," Proc. 2017 3rd IEEE Int. Conf. Sensing, Signal Process. Secur. ICSSS 2017, pp. 301-307, 2017.

[8] H. Setiawan and E. M. Yuniarno, "Biometric Recognition Based on Palm Vein Image Using Learning Vector Quantization," Proc. 2017 5th Int. Conf. Instrumentation, Commun. Inf. Technol. Biomed. Eng. ICICI-BME 2017, no. November, pp. 95-99, 2018.

[9] Y. Ji, Q. Zhao, S. Bi, and T. Shen, "Apple color automatic grading method based on machine vision," Proc. 30th Chinese Control Decis. Conf. CCDC 2018, pp. 56715675, 2018.

[10] Priyanto Hidayatullah, Pengolahan Citra Digital Teori dan Aplikasi Nyata, First Edit. Bandung, 2017.

[11] C. Wang, H. Zhang, and C. Yu, "Research on color recognition of urine test paper based on learning vector quantization (LVQ)," Proc. 2012 2nd Int. Conf. Instrum. Meas. Comput. Commun. Control. IMCCC 2012, pp. 850-853, 2012.

[12] M. Sardogan, A. Tuncer, and Y. Ozen, "Plant Leaf Disease Detection and Classification Based on CNN with LVQ Algorithm," UBMK 2018 - 3rd Int. Conf. Comput. Sci. Eng., pp. 382-385, 2018.

[13] H. Liu, N. Stoll, S. Junginger, and K. Thurow, "Human face orientation recognition for intelligent mobile robot collision avoidance in laboratory environments using feature detection and LVQ neural networks," 2015 IEEE Int. Conf. Robot. Biomimetics, IEEE-ROBIO 2015, pp. 2003-2007, 2015.

[14] P. Li, Y. Zhang, C. Wang, and S. Li, "Surface targets recognition method based on LVQ neutral network," 2015 IEEE Int. Conf. Mechatronics Autom. ICMA 2015, pp. 676-680, 2015.

[15] P. Melin, J. Amezcua, F. Valdez, and O. Castillo, "A new neural network model based on the LVQ algorithm for multi-class classification of arrhythmias," Inf. Sci. (Ny)., vol. 279, no. April, pp. 483-497, 2014. 